Apidologie, 1977, 8 (2), 147-154.

\title{
L'ACCLIMATATION DES TRIGONES AFRICAINES (APIDAE, TRIGONINI) EN FRANCE
}

\author{
Die Akklimatisation der afrikanischen Hypotrigonen \\ (Apidae, Trigonini) in Frankreich
}

\begin{abstract}
R. DARCHEN
Station biologique,

Université $P$, et M. Curie, Paris VI

24620 Les Eyzies

SUMMARY

the acclimatization of Hypotrigona (Apidae, Trigonini)

IN THE SOUTH-WEST OF FRANCE

A population of Hypotrigona, captured in the Ivory Coast, begins its fourth year of life

Breeding techniques are described. This should help specialists to select, for future research, those species which are best adapted to our climate.

\section{RÉSUMÉ}

Une population d'Hypotrigona entame sa quatrième année de vie dans le Sud-Ouest de la France. Elle a cependant déjà hiverné 4 fois. La colonie a été ramenée de la Côte d'Ivoire et installée dans une serre climatisée avec son orifice de sortie en communication permanente avec l'extérieur. Elle arrive done à amasser suffisamment de réserves pour survivre pendant
\end{abstract} in France. It has overwintered 4 times. les périodes froides de l'année.

\section{HISTORIQUE}

L'introduction et l'élevage des Mélipones (Trigones et Mélipones sensu stricto) en France remonte à plus d'un siècle! Les premières tentatives signa- 
lées par Blanchard en 1849, reviennent à une femme qui amena avec elle, en bateau, deux colonies de Rio de Janeiro à Paris, rue Saint-Lazare. Les abeilles ne vécurent que quelques semaines. D'autres colonies arrivèrent ensuite en France régulièrement : la plupart d'entre elles furent installées au Jardin des Plantes. Devant l'échec de tous les essais d'acclimatation, le 7 juin 1872, sous l'impulsion de Raveret Watter, la Société Impériale d'Acclimatation de Paris proposa un prix de 500 francs or à la personne capable de maintenir en vie pendant deux années complètes une colonie d'abeilles américaines, dites sans dard. L'offre était tentante et bien des amateurs ou des professionnels se mirent au travail. Tous échouèrent encore. En 1875, E. Drory importa une quarantaine de colonies envoyées par L. BRUnet, Administrateur Général de l'École Agricole de San Bento au Brésil, et établit ses colonies dans le midi de la France.

Raveret Wattel s'appuya sur ses nombreuses observations pour publier son rapport sur les Mélipones. Devant les échecs, dûs surtout aux difficultés de l'hivernage, la société fut obligée de renouveler plusieurs fois son concours et d'inclure dans la compétition des abeilles venues d'Afrique.

Ce n'est qu'en 1895, vingt ans plus tard, que Perez vit ses efforts couronnés de succès en maintenant des Trigones (Trigona clavipes F.) en vie, trois années consécutives. Cela montre combien l'entreprise était difficile à l'époque. Malheureusement pour nous, cet éleveur chanceux n'a pas indiqué ses conditions d'élevage.

\section{Essais d'acclimatation et condition d'élevage d'une colonie de Trigona} (Hypotrigona)

Au mois de juillet 1972, nous avons récolté une colonie d'Hypotrigones. Elle était installée dans une branche creuse de Crossopterix febrifuga dans une savane à rôniers (Borassus aethiopum) de Côte d'Ivoire. Cette colonie ramenée en France à cette époque vit encore en ce mois de septembre 1976. Elle a donc survécu à quatre hivers périgourdins.

La colonie a été installée dans une serre climatisée dont la température moyenne a oscillé autour de $25^{\circ}$ en 1972 et 1973 , et autour de $20^{\circ}$ les hivers suivants; l'humidité relative est de $75 \%$ environ. Une vitre de la serre a été remplacée par une planche au travers de laquelle passe un tube de verre qui relie la ruche à l'extérieur. Le morceau de bois contenant la ruche bénéficie du climat de la serre, mais les abeilles ne peuvent y voler vu que leur sortie est obligatoirement dirigée vers l'extérieur.

Le laboratoire où est conduit cet élevage est situé dans le Sud-Ouest de la France, aux Eyzies. Ses coordonnées sont les suivantes $0^{0}$ de longitude, $45^{\circ}$ de latitude N. L'altitude est de 67 mètres. Ajoutons que cette Station 


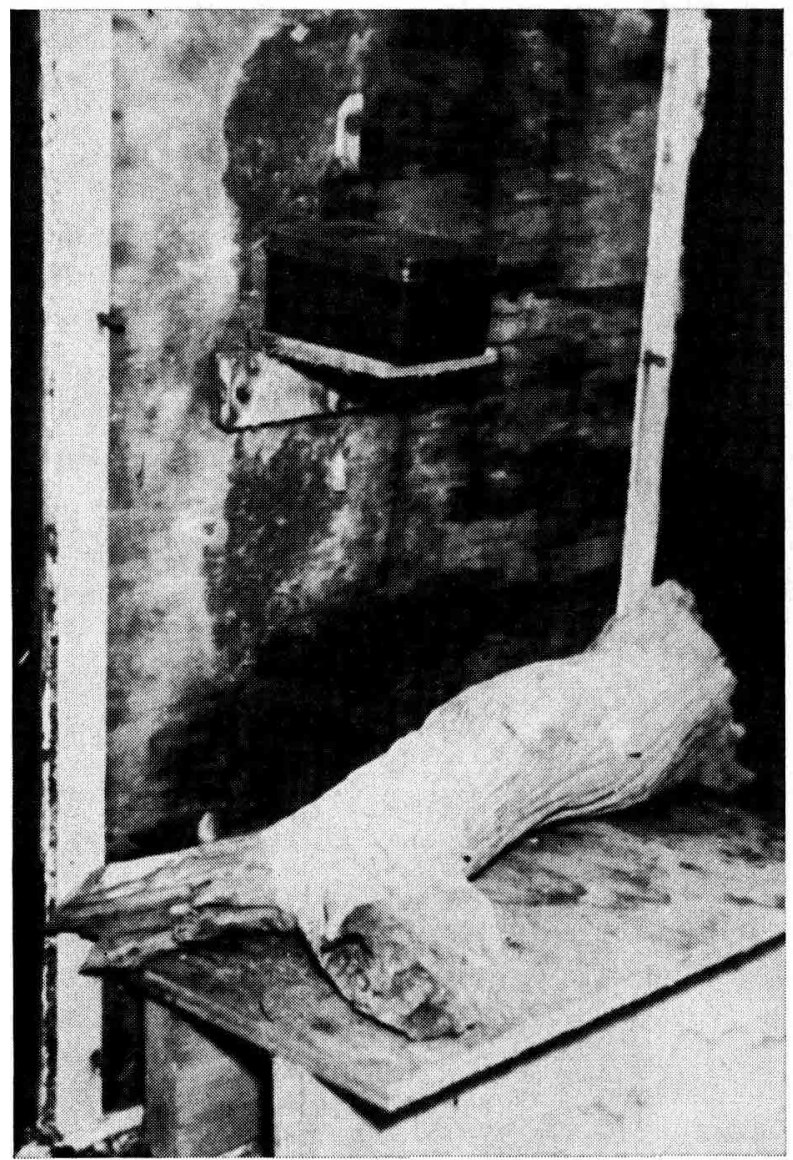

Fig, 1. - Intêrieur de la serre climatisée.

En bas, la branche creuse de Crossopterix est habitée par la colonie d'Hypotrigona. En haut, une petite boîte en plexiglas colorée en bleu foncé contient une nouvelle colonie d'abeilles de la même espèce originaire de Côte d'Ivoire. L'orifice de sortie des ruches est en communication permanente avec l'extérieur de la serre.

Aвв. 1. - Inneres des Hlimatisierten Gewächshauses.

Unten : der hohle Ast von Crossopterix ist von dem Hypotrigona- Volk bewohnt. Oben : ein kleiner, dunkelblau gefürbter Kasten aus Plexiglas enthält ein neues, von der Elfenbeinküste stammendes Bienenvolk der gleichen Art. Das Flugloch der Bienenwohnungen steht ständig mit der Aussenwelt in Verbindung. 
Biologique est à environ $130 \mathrm{~km}$ de Bordeaux. Cette remarque a son importance car la plupart des élevages de Mélipones ont été tentés dans le bordelais dès 1873. Drory n'avait pas moins de 21 colonies de Méliponides de 11 espèces différentes! Il en reçut d'autres les années suivantes. Toutes, sauf deux, moururent durant l'hiver qui suivit. Les survivantes dépérirent très rapidement. Bref, ce fut un échec. Cependant, dans le Sud-Ouest de la France, la technique d'acclimatation semble assez simple, du moins pour les espèces qui ont un minimum de capacités thermorégulatrices. Or, nous savons par expérience, que toutes ne sont pas également douées à cet égard. Les Hypotrigones que nous élevons se rencontrent dans la plus grande partie de l'Afrique tropicale et équatoriale : on les trouve à la fois dans les forêts profondes et humides et dans les savanes beaucoup plus chaudes et plus sèches. Leurs ancêtres ont été retrouvés dans l'ambre de la Baltique. Grâce à son ubiquité naturelle, ce genre Hypotrigona présente donc des prédispositions à l'acclimatation. Drory, malgré ses échecs, nous a montré qu'il avait eu l'intuition que les Mélipones trouveraient dans le Sud-Ouest de la France un climat favorable à leur prospérité. Malheureusement cet auteur, n'ayant pas étudié de près l'écologie et la biologie de ces insectes, ne pouvait savoir qu'il fallait respecter certaines règles, indispensables à la réussite de l'élevage.

\section{DISCUSSION}

Puisque ma colonie est arrivée en 1972 et qu'elle vit encore en 1976, j'ai établi un premier tableau récapitulatif des moyennes de température maximum et minimum de ces 5 années (tabl. 1) et un autre qui indique les minimum des températures absolues durant la même période (tabl. 2).

TABL. 1. - Moyennes mensuelles des températures maximum et minimum de 5 années (janvier 1972 à avril 1976) aux Eyzies (France).

TAB. I. - Monatlicher Durchschnitt der Höchst- und Tiefsttemperaturen während fünf Jahren (Januar 1972 bis April 1976) in Eyzies, Frankreich.

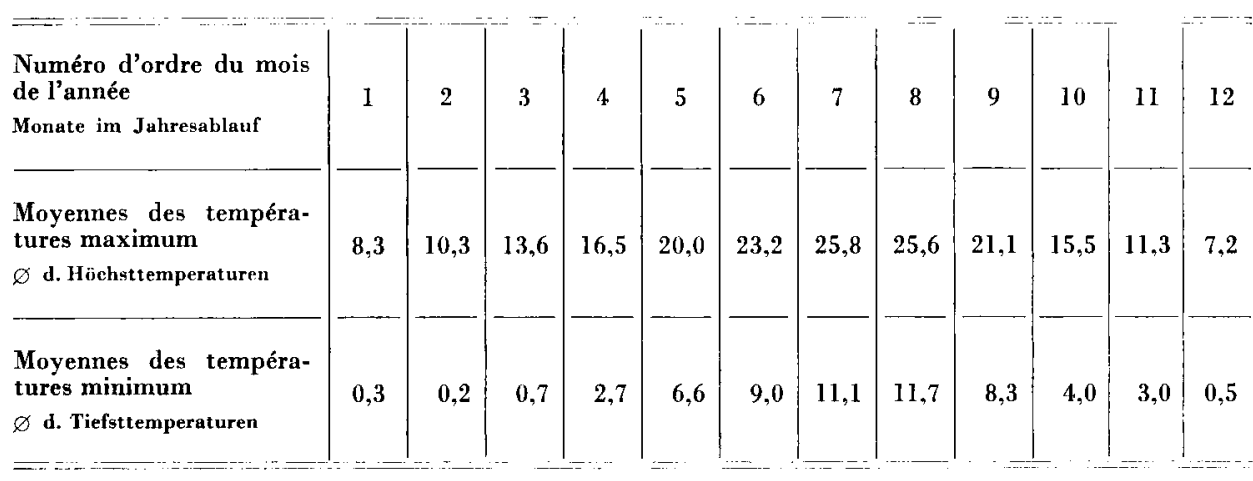


TRIGONES AFRICAINES EN FRANCE

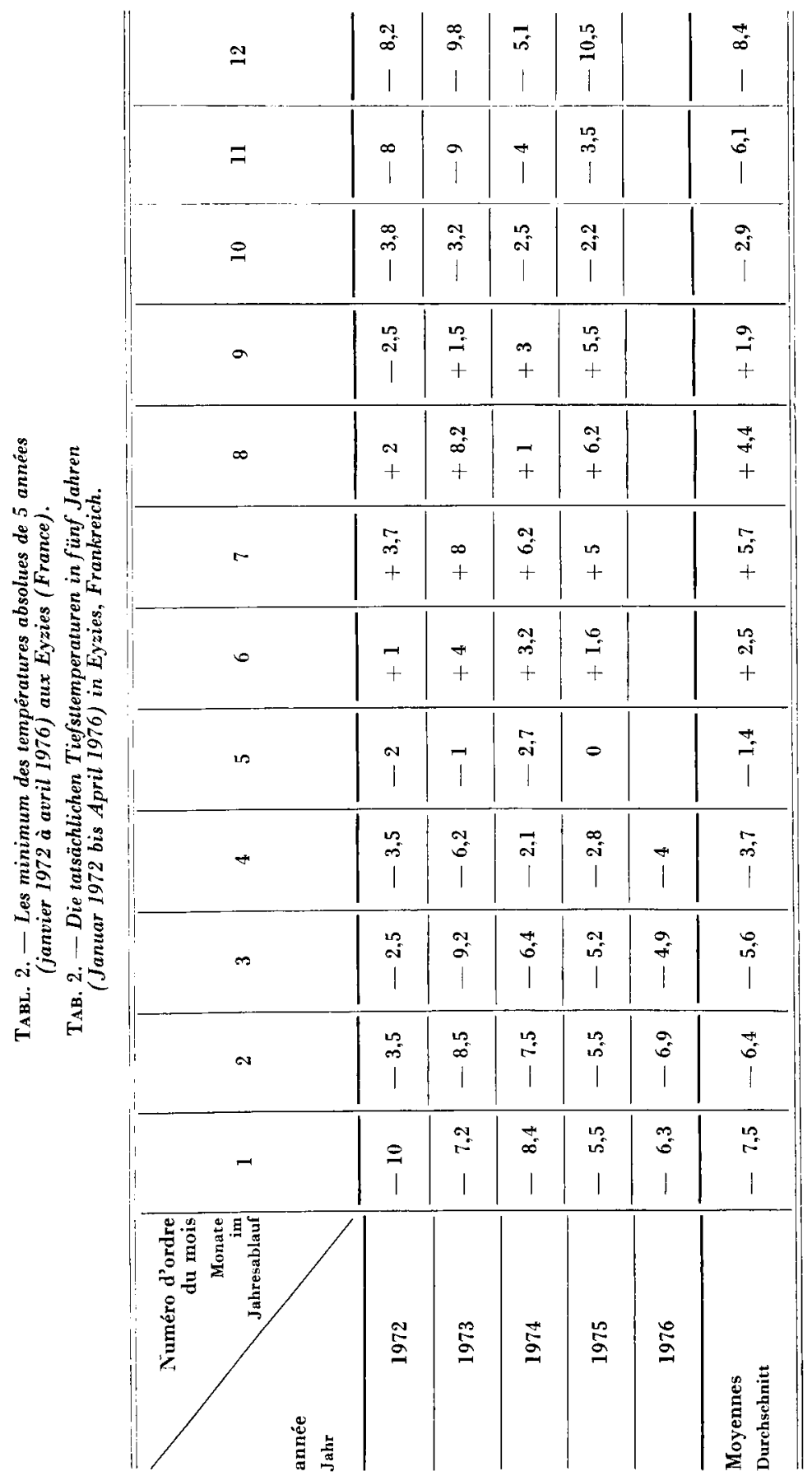


A titre indicatif, et afin de comprendre le succès de mon élevage, je présente deux tableaux ( 3 et 4 ) à peu près semblables aux précédents en utilisant les données de Lecordier sur le climat de la région de Lamto (Côte d'Ivoire) d'où proviennent les abeilles.

TABL. 3. - Moyennes mensuelles des températures maximum et minimum de 11 années à Lamto (Côte d'Ivoire) (janvier 1962 à décembre 1972).

ТАв. 3. -- Monatlicher Durchschnitt der Höchst- und Tiefsttemperaturen während elf Jahren in Lamto, Elfonbeinküste. (Januar 1962 bis Dezember 1972).

\begin{tabular}{l|c|c|c|c|c|c|c|c|c|c|c|c}
\hline \hline $\begin{array}{l}\text { Numéro d'ordre du mois } \\
\text { de l'année } \\
\text { Monate im Jahresablauf }\end{array}$ & 1 & 2 & 3 & 4 & 5 & 6 & 7 & 8 & 9 & 10 & 11 & 12 \\
\hline $\begin{array}{l}\text { Moyennes des tempéra- } \\
\text { tures maximum } \\
\varnothing \text { der Höchsttemperaturen }\end{array}$ & 33,6 & 35,5 & 35,2 & 34,3 & 33,5 & 31,3 & 30,3 & 30,1 & 31,3 & 32,5 & 33,2 & 32,8 \\
\hline $\begin{array}{l}\text { Moyennes des tempéra- } \\
\text { tures minimum } \\
\varnothing \text { der Tiefsttemperaturen }\end{array}$ & 20,7 & 22,1 & 22,6 & 22,5 & 22,5 & 22,1 & 21,6 & 21,4 & 21,8 & 22,1 & 21,8 & 21,3 \\
\hline
\end{tabular}

TaBL. 4. - Les valeurs absolues mensuelles des minimum et maximum de température de 11 années (janvier 1962 à décembre 1972) à Lamto (Côte d'Ivoire).

TАв. 4. - Die tatsächlichen monatichen Tiefst- u. Höchsttemperaturen während elf Jahren (Januar 1962 bis Dezember 1972) in Lamto, Elfenbeinkïste.

\begin{tabular}{|c|c|c|c|c|c|c|c|c|c|c|c|}
\hline $\begin{array}{l}\text { Numéro d'ordre du mois } \\
\text { Monate im Jahresablauf }\end{array}$ & 1 & 2 & 3 & 4 & 5 & 6 & 7 & 8 & 9 & 10 & 11 \\
\hline $\begin{array}{l}\text { Valeurs minimum } \\
\text { Tiefsttemperaturen }\end{array}$ & 12,2 & 13,9 & 19,9 & 20,0 & 20,2 & 19,1 & 17,8 & 16,8 & 17,0 & 19,8 & 18,0 \\
\hline $\begin{array}{l}\text { Valeurs maximum } \\
\text { Höchsttemperaturen }\end{array}$ & 21,6 & 22,6 & 22,6 & 22,6 & 23,1 & 22,4 & 22,1 & 21,7 & 22,1 & 22,3 & 21,8 \\
\hline $\begin{array}{l}\text { Moyennes } \\
\text { des valeurs absolues } \\
\varnothing \text { der tatsächlichen Tempe- } \\
\text { raturen }\end{array}$ & 17,3 & 19,1 & 21,1 & 20,9 & 21,1 & 20,7 & 20,2 & 19,5 & 20,2 & 20,8 & 20,6 \\
\hline
\end{tabular}

L'étude comparative des tableaux 1 et 3 et des tableaux 2 et 4 nous permet de définir les premières règles d'élevage de certaines espèces de Trigones, dans nos régions tempérées d'Europe. 
1) Il est clair que les Hypotrigones des savanes de Côte d'Ivoire s'accommodent de températures extérieures élevées (autour de $35^{\circ}$ ) qui sont souvent atteintes aux Eyzies car, durant les mois les plus chauds, la température moyenne maximum se tient autour de $25^{\circ}$. Il n'y a done pas pour les colonies importées de problèmes de régulation thermique insurmontables durant les fortes chaleurs de l'été périgourdin.

2) Nous avons observé en Afrique que les colonies d'Hypotrigones prospéraient pendant les mois de février, août et décembre bien qu'à cette époque la température moyenne oscille autour de $19^{\circ}$ et même $17^{\circ}$ durant le mois de janvier (tabl. 4); aux Eyzies au cours de la journée, pendant 6 mois de l'année, de mai à octobre compris, la température moyenne ne descend pas audessous de cette moyenne africaine des mois les plus froids (tabl. 1).

3) Ces Hypotrigones supportent même un climat dont la température peut descendre au-dessous de $12^{\circ}$ sans que meure le jeune couvain, comme le témoignent nos analyses de population sur place. Nous savons, en outre, que les adultes survivent pendant plusieurs heures, sinon plusieurs jours à des températures situées au-dessous de $10^{\circ}$ mais, dans ce cas, les plus jeunes larves meurent dans les cellules. Il faudrait d'ailleurs effectuer des expériences systématiques pour déterminer les températures léthales pour les populations en équilibre.

Il est done clair maintenant que les colonies d'Hypotrigones peuvent survivre facilement dans le Sud-Ouest de la France à condition d'assurer à ces abeilles au moins un minimum de sécurité de $17^{0}$ pendant l'hiver et les nuits froides des autres saisons. Dans ces conditions, ces abeilles ont le temps nécessaire pour constituer leurs réserves de nourriture et faire face à l'hivernage qui ne dure que 3 à 4 mois, aux Eyzies.

\section{CONCLUSION}

La réussite de notre élevage d'Hypotrigones en France nous incite à tenter l'introduction d'autres espèces africaines ou américaines.

Depuis plus de 10 ans nous avons l'expérience de l'élevage des abeilles tropicales. Les essais d'acclimatation en Europe, que nous espérons tenter dorénavant, porteront de préférence sur des insectes vivant soit dans les savanes, soit, pour l'Amérique, dans des régions montagneuses. En effet, les animaux issus de ces régions sont soumis à des variations climatiques souvent très importantes et sont mieux aptes à résister sous nos climats. Au contraire, les insectes habitant les régions côtières ou les forêts humides ont des capacités thermorégulatrices moindres et leur maintien en France est beaucoup plus aléatoire. La réussite de ces élevages permet d'envisager des 
expériences qui, jusqu'ici, m'ont été impossibles à tenter dans les pays d'origine de ces insectes, faute d'un équipement technique adéquat, en particulier une analyse affinée du déterminisme des castes chez les Trigones.

Reçu pour publication en fêvrier 1977.

Eingegangen im Februar 1977.

\section{ZUSAMMENFASSUNG}

Ein Volk von Hypotrigona sp. beginnt nunmehr sein viertes Lebensjahr im Südwesten Frankreichs und hat inzwischen viermal hier überwintert. Das Volk wurde von der Elfenbeinküste hierhergebracht und in einem klimatisierten Gewächshaus so aufgestellt, dass es durch das Flugloch ständig mit der Aussenwelt in Berührung kam. Demnach ist es ihm gelungen, genügend Vorräte zu sammeln, um die hiesige kalte Jahreszeit zu überleben.

Vier Tabellen, die das Klima der Savanne, der Heimat der Bienen und das von Eyzies, Frankreich, in der Umgebung des Laboratoriums aufzeigen, ermöglichen es 1. unseren Aufzuchterfolg zu verstehen; 2. die Aufzuchtbedingungen dieser Insekten im Südwesten Frankreichs festzulegen und 3. die Bienenforscher bei der Wahl der in Europa aufzuziehenden Arten zu beraten.

\section{RÉFÉRENCES BIBLIOGRAPHIQUES}

Blanchand C. E., 1849. - Melipona, Méliponites. In d'Orbigny, Dictionnaire d'Histoire Naturelle, Paris, 8, 85-89.

Darchen R., 1969. - La biologie des Trigones et des Mélipones (Hyménoptères, Apidae) à la lumière des travaux récents. Ann. Biol., 8, 455-490.

Darchen R., 1973. - La thermorégulation et l'écologie de quelques espèces d'abeilles sociales d'Afrique (Apidae, Trigonini) et Apis mellifica var. Adansonii. Apidologie, 4, 341-370.

Darchen R., 1976. - L'élevage d'Apis mellifica adansonii dans le Sud-Ouest de la France. Gaz. apic., 77 (1), 222-224.

Drory E., 1873. - Note sur quelques espèces de mélipones de l'Amérique du Sud. Act. Soc. Lin., Bordeaux, 29, 31-33.

Lecordier CH., 1974. - Le climat. I. Les facteurs physiques du milieu. Bull. Liaison Lamto, Num. Spécial, 1, 45-104.

Perez J., 1895. - Sur la production des femelles et des mâles chez les méliponites. C.R. Arcad. Sci. Paris, 120, 273-275. 